\title{
Zipper-interacting protein kinase interacts with human cell division cycle 14A phosphatase
}

\author{
WEI WU ${ }^{1 *}$, HAIYING HU²* ${ }^{2}$ ZI YE ${ }^{1}$, MANCHEONG LEONG ${ }^{3}$, MIN HE ${ }^{1}$, \\ QIN $\mathrm{LI}^{4}$, RENMING HU ${ }^{1}$ and SHUO ZHANG ${ }^{1}$

\begin{abstract}
${ }^{1}$ Department of Endocrinology and Metabolism, Institute of Endocrinology and Diabetology at Fudan University, Huashan Hospital, Fudan University, Shanghai 200040; ${ }^{2}$ Department of Endocrinology and Metabolism, International Healthcare Center, Second Affiliated Hospital of Zhejiang University, Hangzhou, Zhejiang 310009

${ }^{3}$ Department of Endocrinology, City University of Macau, Macau 000853; ${ }^{4}$ Department of Endocrinology, Shanghai Tenth People's Hospital, School of Medicine, Tongji University, Shanghai 200000, P.R. China
\end{abstract}

Received February 13, 2014; Accepted November 4, 2014

DOI: $10.3892 / \mathrm{mmr} .2014 .3067$

\begin{abstract}
Zipper-interacting protein kinase (ZIPK) is a novel serine/threonine protein kinase and a member of a large family of protein kinases, known as the death-associated protein kinases. However, the function of ZIPK has yet to be fully elucidated, as few physiological substrates have currently been identified. In the present study, a yeast two-hybrid screen was used and the human cell division cycle 14A (HsCdc14A) phosphatase was identified as a novel ZIPK binding protein. To the best of our knowledge, this is the first study to report the interaction between these proteins. The interaction between ZIPK and HsCdc14A was confirmed by in vitro experiments. In addition, ZIPK-mediated phosphorylation was shown to activate the phosphatase activity of $\mathrm{HsCdc14A}$. These findings indicated that ZIPK may also be involved in the regulation of the cell cycle in human cells, by interacting with HsCdc14A.
\end{abstract}

\section{Introduction}

Zipper-interacting protein kinase (ZIPK) is a novel serine/threonine (Ser/Thr) protein kinase, which was initially cloned and identified in $1998(1,2)$. ZIPK is a member of the large family of death-associated protein kinases and has been associated with the regulation of numerous cellular processes, including cell death (1), cell motility (3) and mitotic processes (4), as well as smooth muscle contraction $(5,6)$. In addition, ZIPK

Correspondence to: Mr. Renming $\mathrm{Hu}$ or Miss. Shuo Zhang, Department of Endocrinology and Metabolism, Institute of Endocrinology and Diabetology at Fudan University, Huashan Hospital, Fudan University, 12 Middle Wulumugi Road, Shanghai 200040, P.R. China

E-mail: renminghu@fudan.edu.cn

E-mail: colleen0708@hotmail.com

*Contributed equally

Key words: zipper-interacting protein kinase, human cell division cycle $14 \mathrm{~A}$, cell cycle, interaction interacts with the signal transducer and activator of transcription 3 (STAT3), a latent cytoplasmic transcription factor that plays a role in cell growth and apoptosis. ZIPK has been shown to phosphorylate STAT3 on Ser-727, thus enhancing the transcriptional activity of STAT3 (7). Vetterkind et al (8) have demonstrated that prostate apoptosis response-4 (Par-4), which is characterized mainly as a proapoptotic protein, targets ZIPK to the cytoskeleton in nonmuscle cells, leading to apoptosis. By contrast, in smooth muscle cells, Par-4 supports contractility by targeting ZIPK to the cytoskeleton (9). A previous study, conducted by the authors of the present study, demonstrated that incubation of smooth-muscle cells with glucose induced a time- and dose-dependent increase in the protein expression levels of ZIPK (unpublished data). These findings indicate that ZIPK plays a key role in cellular function.

However, only a limited number of studies have focused on the effects of ZIPK on cell death, motility and mitotic processes. To date, the interacting partners of ZIPK, particularly those associated with the cell cycle, have been rarely identified and the regulatory network of ZIPK is not fully understood. The aim of the present study was to investigate the interaction of ZIPK with the human cell division cycle $14 \mathrm{~A}$ (HsCdc14A) phosphatase in vitro, and identify whether ZIPK plays a role in the cell cycle regulation.

\section{Materials and methods}

Yeast two-hybrid assay. The study was approved by the ethics committee of Huashan Hospital, Fudan University (Shanghai, China). Yeast two-hybrid interaction screening was performed as described in previous studies (10-12). Full-length HsCdc14A was introduced into the GAL4 DNA (Clontech Laboratories, Inc., Mountain View, CA, USA) binding domain as a bait. Its interaction partner was a human testis cDNA library (Clontech Laboratories, Inc.) integrated with GAL4 transactivation domain. The bait plasmids and the cDNA library plasmids were used to transform a yeast strain (AH109; Clontech Laboratories, Inc.), containing HIS3 and LacZ reporter genes, using a lithium acetate method (13). The 
transformed samples were spread onto plates with a synthetic defined (SD)/Leu/Trp/His medium. Subsequently they were spread onto a SD/-Leu/-Trp/-His/-Ade/X- $\alpha$-Gal medium for further selection. Positive clones (those with diameter $>3 \mathrm{~mm}$ ) were co-transformed into the AH109 yeast with bait plasmids. Following extraction of plasmids from yeast, the plasmids were used to transform Escherichia coli (Tiandz, Inc., Beijing, China). The interacting protein was verified by sequencing the plasmid following extraction from Escherichia coli.

Glutathione S-transferase (GST) pull-down assay. HsCdc14A cDNA [full length, amino acid (aa) 1-623; deletion mutant, aa1-348N terminal; or aa349-623C terminal] was fused with a 6-His tag (pET-28a; Tiandz Inc., Beijing, China). ZIPK cDNA (full length, aa1-454) was cloned into a pGEX-5X-3 vector (Amersham Biosciences, Piscataway, NJ, USA). Using a previously described method (11), these proteins were expressed in Escherichia coli BL21 (DE3) cells (Tiandz Inc.), and purified by $\mathrm{Ni}^{2+}$ nitrilotriacetic acid (for aa1-348; Sigma-Aldrich, St. Louis, MO, USA) or glutathione beads (for aa349-623; Sigma-Aldrich). An in vitro pull-down assay was performed using purified GST-fused ZIPK and His-tagged HsCdc14A with phosphate-buffered saline (PBS), containing 0.1\% Triton X-100 (Sangon Biotech, Shanghai, China) for $4 \mathrm{~h}$ at $4^{\circ} \mathrm{C}$. The beads were washed three times with PBS containing $1 \%$ Triton X-100, and once with PBS alone. The beads were then boiled in SDS-PAGE sample buffer for $5 \mathrm{~min}$ and used for western blot analysis.

Co-immunoprecipitation. Human embryonic kidney (HEK) 293T cells from the American Type Culture Collection (Manassas, VA, USA) were grown to 50-60\% confluence in Dulbecco's modified Eagle's medium (Hyclone, Logan, UT, USA), supplemented with $10 \%$ fetal bovine serum (Hyclone), at $37^{\circ} \mathrm{C}$ in an atmosphere containing $5 \% \mathrm{CO}_{2}$. The cells were then co-transfected with green fluorescent protein (GFP)-HsCdc14A (full length or deletion mutant aa1-348N-terminal or aa349-623 C-terminal) and FLAG-ZIPK using the standard $\mathrm{CaCl}_{2}$ method (14), with a GFP-transfected plasmid as a control. $36 \mathrm{~h}$ later, the cells were collected and lysed in lysis buffer (50 mM HEPES, pH 7.2; $150 \mathrm{mM} \mathrm{NaCl} ; 2 \mathrm{mM}$ ethylene glycol tetraacetic acid; and $0.1 \%$ Triton X-100), together with protease inhibitor mixture (Aprotinin, Bestatin and Leupeptin Pepstatin A; Sigma-Aldrich). The lysate was then clarified using centrifugation at $16,000 \mathrm{x} \mathrm{g}$ for $10 \mathrm{~min}$ at $4^{\circ} \mathrm{C}$. The cell lysate was incubated with anti-FLAG antibody conjugated to agarose beads (Sigma-Aldrich) for $4 \mathrm{~h}$ at $4^{\circ} \mathrm{C}$, and then was washed five times using lysis buffer. The proteins were boiled for $5 \mathrm{~min}$ and used for subsequent western blot analysis.

Western blotting analysis. Following SDS-PAGE using a $12 \%$ gel, proteins were transferred onto poly vinylidene difluoride membranes, which had been obtained from Millipore Corporation (Billerica, MA, USA). The membranes were incubated with primary antibodies overnight at $4^{\circ} \mathrm{C}$ with gentle shaking (Qite Analytical Instrument Co.Ltd., Shanghai, China). Membranes were then incubated with secondary antibodies at room temperature for $2 \mathrm{~h}$. Immunoreactive signals were detected using an enhanced chemiluminescence
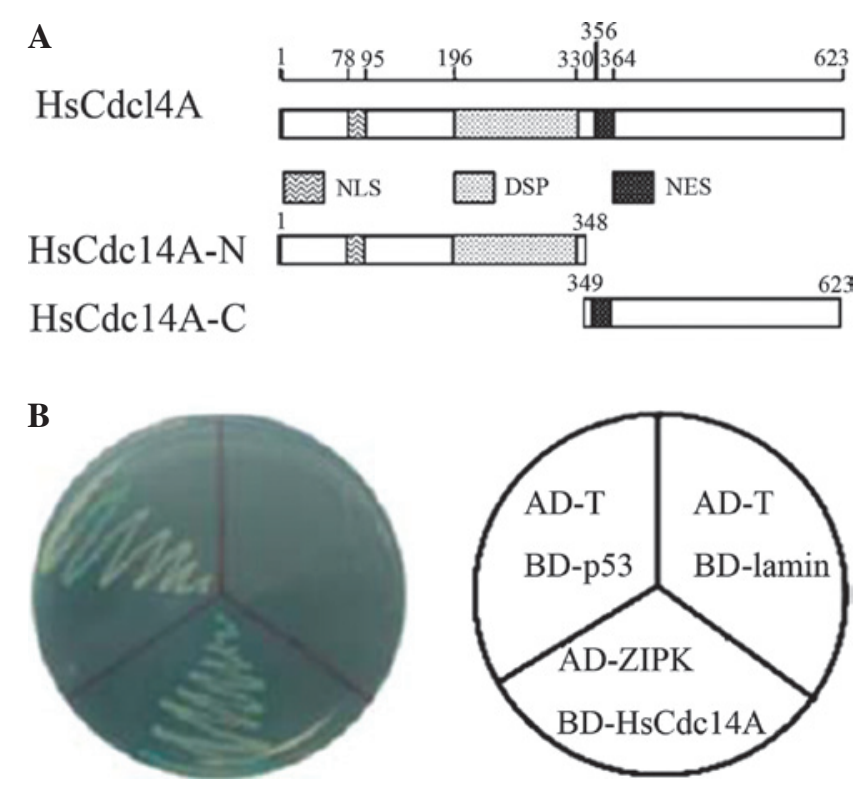

Figure 1. Yeast two-hybrid screening identified HsCdc14A as a ZIPK-binding protein. Schematic diagrams of (A) HsCdc14A protein structure, containing the DSP, NLS and NES domains; and (B) the interaction of ZIPK with HsCdc14A in AH109 yeast cells. BD-p53 and AD-T were co-transformed as the positive controls, whereas BD-lamin and AD-T were the negative controls. HsCdc14A, human cell division cycle 14A; ZIPK, zipper-interacting protein kinase; HsCdc14A-N, N-terminus; HsCdc14A-C, C-terminus; DPS, dual-specificity phosphatase; NLS, nuclear localization signal; NES, nuclear export signal.

kit (Pierce Chemical, IL, USA) and visualized by autoradiography on Kodak BioMAX film (Kodak, Rochester, NY, USA). The following antibodies were used: Mouse monoclonal anti-His antibody (1:1,000; Cell Signaling Technology, Inc., Danvers, MA, USA); rabbit polyclonal anti-GFP antibody (1:1,000; Cell Signaling Technology, Inc); mouse monoclonal anti-FLAG antibody (1:2,000; Sigma-Aldrich); and anti-rabbit $(1: 2,000)$ and anti-mouse $(1: 2,000)$ horseradish peroxidase-conjugated secondary antibodies (Cell Signaling Technology, Inc.).

Cell cycle analysis. A cell cycle analysis was conducted as previously described (15). Briefly, HEK293T cells were grown in six-well plates with density $\sim 1 \times 10^{6}$ cells per well and transfected with the following plasmids: GFP, GFP-HsCdc14A, GFP-HsCdc14A N-terminus, GFP-HsCdc14A C-terminus, GFP-ZIPK, GFP-HsCdc14A+ and GFP-ZIPK. $72 \mathrm{~h}$ later, cells were collected and washed with ice cold PBS. Next, they were fixed with $70 \%$ ethanol for $\geq 1 \mathrm{~h}$. The fixed cells were then washed and stained with propidium iodide (PI; Invitrogen Life Technologies, Carlsbad, CA, USA) for $1 \mathrm{~h}$ at room temperature. Subsequently, the PI-stained nuclei were analyzed using the BD FACSCalibur ${ }^{\mathrm{TM}}$ system (BD Biosciences, Franklin Lakes, NJ, USA).

Apoptosisassay. Annexin V-fluorescein isothiocyanate (FITC)/ PI staining using an Apoptosis Detection Kit (Invitrogen, Life Technologies) was used in order to detect apoptotic cells. HEK293T cells were collected at $72 \mathrm{~h}$ post-transfection with the plasmids listed above. Cells were then trypsinized and collected by centrifugation at $300 \mathrm{x}$ g for $5 \mathrm{~min}$. Cells were washed once with PBS, resuspended in 1X binding buffer and 
A

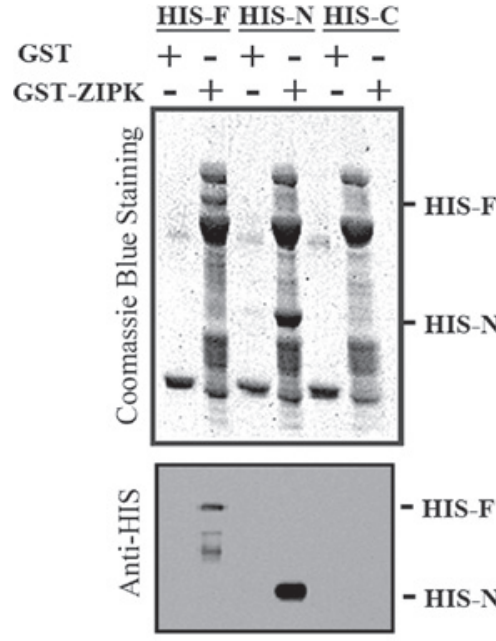

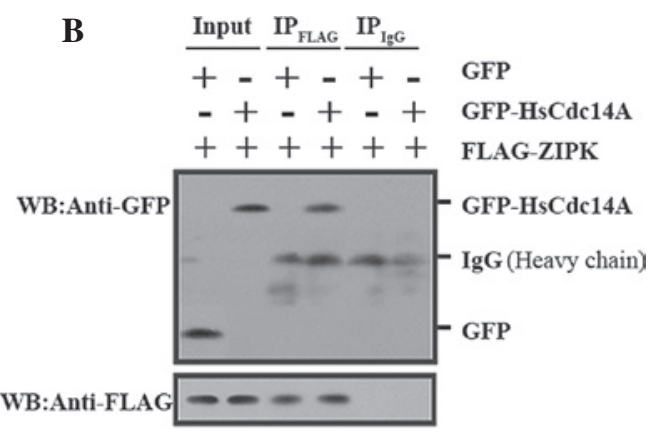

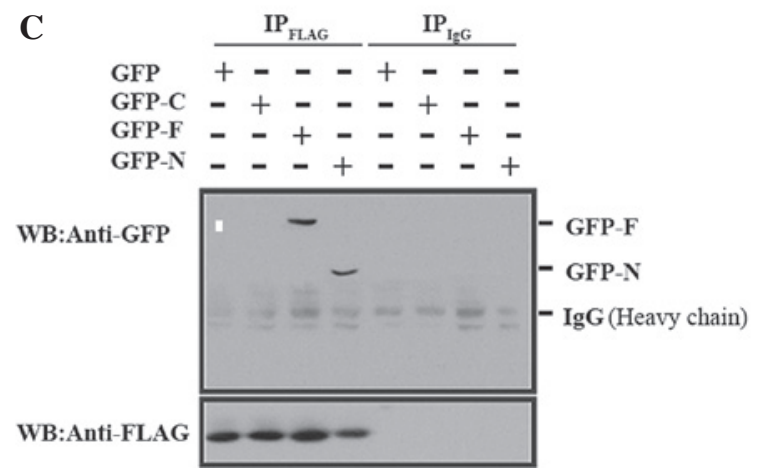

Figure 2. ZIPK interaction with HsCdc14A N-terminus (aa 1-348). (A) His-HsCdc14A and its N terminus were pulled down by GST-ZIPK. Three different deletions of HsCdc14A (His-F, aa 1-623; His-N, aa 1-348; or His-C, aa 349-623) were separately incubated with GST-ZIPK, followed by GST antibody, and were separated with SDS-PAGE. The gels were then stained with Coomassie Blue (upper panel) or analyzed by western blot analysis using anti-HIS (lower panel). The full length and N-terminus HsCdc14A bands interacted with ZIPK, whereas the HsCdc14A C-terminus did not interact with ZIPK (no band). (B) Full length HsCdc14A was co-immunoprecipitated with ZIPK. The cell lysate (input) and immunoprecipitate fractions (IP FLAG $_{\text {and IP }}$ IgG , respectively), obtained from the cell lysates, were immunoprecipitated with anti-FLAG antibodies or non-specific IgG and subjected to an immunoblotting assay with anti-GFP (upper panel) or anti-FLAG (lower panel). (C) HsCdc14A N-terminus was co-immunoprecipitated with ZIPK. The cell lysates (GFP-F, aa 1-623; GFP-N, aa 1-348; or GFP-C, aa 349-623; co-transfected with FLAG-tagged ZIPK) were immunoprecipitated with anti-FLAG or nonspecific IgG, and the immunoprecipitates obtained were analyzed by western blot analysis using anti-GFP (upper panel) or anti-FLAG (lower panel). HsCdc14A, human cell division cycle 14A; ZIPK, zipper-interacting protein kinase; GST, glutathione S-transferase; -F, full length HsCdc14A; -N, N-terminus; -C, C-terminus; aa, amino acid; SDS-PAGE, sodium dodecyl sulfate-polyacrylamide gel electrophoresis; IgG, immunoglobulin G; GFP, green fluorescent protein.

stained with Annexin V-FITC for 15 min. The cell nuclei were then counter-stained with PI in order to detect necrosis. Flow cytometric analysis was performed to analyze the percentage of apoptotic cells, using the BDFACSCalibur ${ }^{\mathrm{TM}}$ system.

Statistical analysis. All experiments were performed at least in triplicate. The results are expressed as the mean \pm standard deviation, and the data were analyzed using Student's t-test to detect statistically significant differences among the groups. $\mathrm{P}<0.05$ was considered to indicate a statistically significant difference. Statistical analyses was performed using SPSS Version 11 (IBM SPSS, Armonk, NY, USA).

\section{Results}

Identification of HsCdc14A as a ZIPK-interacting protein. Nucleotide sequencing was performed in previous experiments using full length HsCdc14A cDNA as a bait in order to screen through a human testis cDNA library. ZIPK was identified as a potential binding protein. Subsequent assays validated that $\mathrm{HsCdc14A}$ can bind to ZIPK (Fig. 1). To the best of our knowledge, the present study reported for the first time a potential interaction between ZIPK and HsCdc14A.

ZIPK and HsCdc14A interactions. Subsequent experiments were conducted to verify the interaction between ZIPK and HsCdc14A. In a GST pull-down assay, GST-ZIPK was found to pull down HsCdc14A, as compared with GST alone (Fig. 2A). Various deletion mutations of $\mathrm{HsCdc14A}$ were constructed to map the interaction fragments of the two proteins. Two deletions of $\mathrm{HsCdc14A}$ were constructed (Fig. 1A). The N-terminus (aa 1-348) consisted of the nuclear localization signal (NLS) and dual-specificity phosphatase (DSP) domains, while the C-terminus (aa 349-623) contained the nuclear export signal (NES) domain. The results of the subsequent GST pull-down experiment indicated that ZIPK interacted directly with the $\mathrm{N}$-terminus of $\mathrm{HsCdc14A}$, but not with the C-terminus of HsCdc14A (Fig. 2A). These results were consistent with the important function of the DSP domain of HsCdc14A.

In order to verify the interaction between ZIPK and HsCdc14A in mammalian cells, HEK 293T cells were transfected with GFP-tagged HsCdc14A or a control plasmid, 


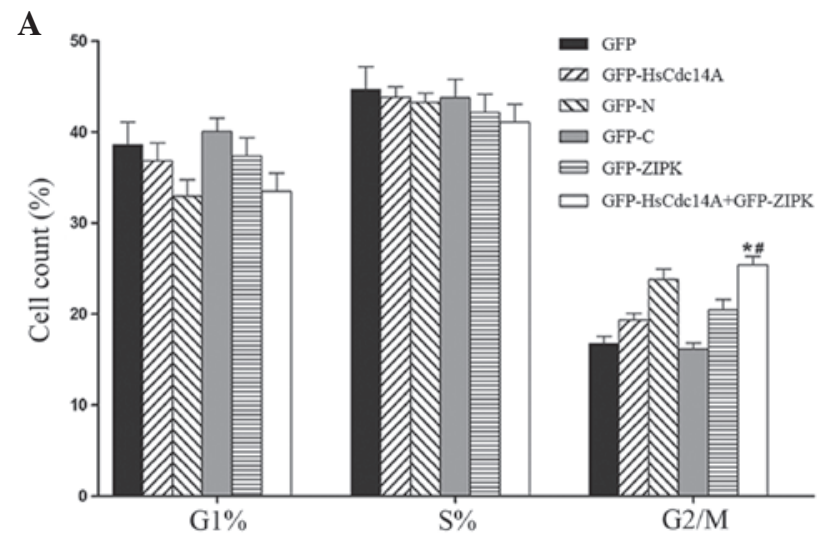

$\mathbf{B}$

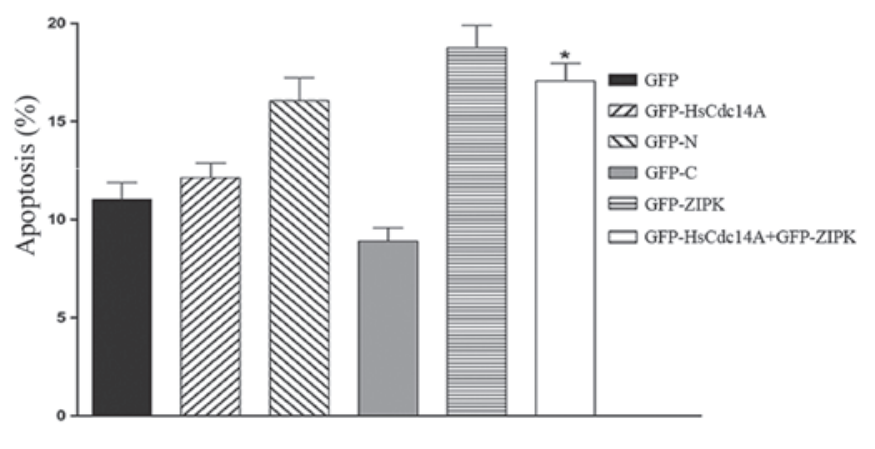

Figure 3. Co-transfection of HEK 293T cells with ZIPK and HsCdc14A affected cell cycle progression and resulted in cell apoptosis. (A) Effect of co-transfected ZIPK and HsCdc14A on the cell cycle progression; the relative number of cells was increased in the $\mathrm{G}_{2} / \mathrm{M}$ phase compared with cells transfected with $\mathrm{HsCdc14} \mathrm{A}$ and was similar to cells transfected with the HsCdc14A N-terminus. (B) Determination of apoptosis by Annexin V assay. A considerable increase was observed in the number of Annexin V-positive cells upon co-transfection with ZIPK and HsCdc14A, compared with the cells transfected with HsCdc14A alone, and was similar to the cells transfected with $\mathrm{HsCdc14 \textrm {A }} \mathrm{N}$-terminus. Three independent experiments were performed for each data point and the data are expressed as the mean \pm standard deviation. ${ }^{*} \mathrm{P}<0.05$, vs. GFP-HsCdc14A group; ${ }^{~} \mathrm{P}<0.05$, vs. GFP-ZIPK group. ZIPK, zipper-interacting protein kinase; HsCdc14A, human cell division cycle 14A; GFP, green fluorescent protein; -N, N-terminus; -C, C-terminus.

along with FLAG-tagged ZIPK. The cell lysates were immunoprecipitated with anti-FLAG antibodies. Subsequently, the obtained immunoprecipitates were analyzed by western blotting using an anti-GFP antibody, in order to detect the presence of bound HsCdc14A. FLAG-tagged ZIPK was found to co-precipitate with GFP-tagged HsCdc14A, but not with GFP alone (Fig. 2B).

The regions responsible for the interaction between ZIPK and $\mathrm{HsCdc14} \mathrm{A}$ were also determined. A series of GFP-tagged HsCdc14A (full length; GFP-HsCdc14A N-terminus or C-terminus) were co-transfected into HEK 293T cells with FLAG-tagged ZIPK. Co-immunoprecipitation experiments indicated that ZIPK interacted strongly with the HsCdc14A $\mathrm{N}$-terminus, containing the NLS and DSP domains (Fig. 2C).

Co-transfected ZIPK and HsCdc14A plasmids interfere with mitotic progression and cause apoptosis. Due to the identification of the physical interaction between ZIPK and HsCdc14A, the present study aimed to determine the functional relevance of the ZIPK-HsCdc14A interaction in mitotic progression. HEK 293T cells were separately transfected with various plasmids. As shown in Fig. 3A, the cell cycle assay demonstrated that the $\mathrm{G}_{2} / \mathrm{M}$ phase cell population was significantly increased in cells that were co-transfected with ZIPK and HsCdc14A plasmids, when compared with the cells that were transfected with HsCdc14A alone. These results were similar to those obtained following transfection with the HsCdc14A $\mathrm{N}$ terminus (the phosphatase domain and its activity may be inhibited by the C-terminus). The results indicated that the mitotic progression was arrested in cells co-transfected with ZIPK and HsCdc14A. In addition, the percentage of apoptotic cells following transfection with the various plasmids was determined. A considerable increase was observed in the number of Annexin V-positive cells upon co-transfection with ZIPK and HsCdc14A, compared with the cells transfected with HsCdc14A alone (Fig. 3B). The percentage of apoptotic cells was similar to the cells transfected with the HsCdc14A N-terminus. These results indicated that apoptosis was increased in the cells co-transfected with ZIPK and HsCdc14A. Therefore, the control of HsCdc14A phosphatase activity by ZIPK is hypothesized to play an important role in mitotic progression and cell apoptosis.

\section{Discussion}

ZIPK was initially identified as a Ser/Thr kinase that binds activating transcription factor 4 (ATF4). ATF4 is a member of the activating transcription factor and cyclic adenosine monophosphate-responsive element-binding protein family of transcription factors (1). ZIPK aggregates through its C-terminal leucine zipper structure, thereby becoming an active enzyme. A previous study identified that the ectopic expression of ZIPK in NIH-3T3 murine fibroblast cells induced apoptosis (1). By contrast, the kinase-inactive ZIPK K42A mutant was not found to induce apoptosis, indicating that the catalytic activity of ZIPK stimulates cell apoptosis (1). Numerous kinases that mediate cell growth are known; however, only a few protein kinases associated with apoptosis have been identified, besides ZIPK. Previous studies demonstrated that ZIPK participates in the regulation and possibly the coordination of mitosis and cytokinesis, by interacting with the proapoptotic protein, Par- 4 , and the CDC5 protein $(4,16)$. In addition, ZIPK is a centromere-specific histone kinase that may play a role in the labeling of centromere-specific chromatin for subsequent mitotic processes (17). Furthermore, previous studies have indicated that ZIPK, as a regulator of myosin phosphatase, may play a pivotal role in the regulation of cell motility, reorganization of actin filaments and control of smooth muscle contraction in smooth muscle cells $(4,6,7)$. However, only a few interacting partners of ZIPK have been identified and the regulatory networks of ZIPK remain to be elucidated.

The present study demonstrated that ZIPK interacted with the $\mathrm{HsCdc} 14 \mathrm{~A}$ protein in vitro, playing an important role in the regulation of the cell cycle. The interaction was shown

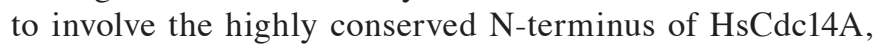


indicating that ZIPK may be involved in the cell cycle regulation.

Cdc14 is a protein phosphatase conserved between yeast and humans (10). Genetic analyses have suggested that the yeast $\mathrm{Cdc14}$ plays a pleiotropic role during the cell cycle, regulating DNA replication and the exit from mitosis by dephosphorylating cyclin-dependent kinase (Cdk) targets (18-20). Mammalian cells express two functional homologs of the yeast Cdc14, known as HsCdc14A and HsCdc14B (21). These two proteins remain poorly understood; however, recent evidence has indicated that they play an isoform-specific role in centrosome separation/maturation and spindle stability, with the possibility of additional role in the mitotic exit and cytokinesis (21). The majority of previous studies have investigated HsCdc14A, which was demonstrated to interact with interphase centrosomes and regulate the centrosome duplication cycle $(22,23)$. In addition, HsCdc14A is located at the central spindle during anaphase, where it appears to be involved in the spatial regulation of the Aurora B kinase, a key regulator of chromosome segregation and cytokinesis (24). HsCdc14A may also regulate p53 and Cdk1/cyclin B; thus, dysregulation of HsCdc14A may play an important role in carcinogenesis (25). Besides p53, a previous study revealed that $\mathrm{HsCdc14A}$ can dephosphorylate the products of Cdk, including hCdh1 and cyclin E (26). However, the mechanism through which the HsCdc14A function is integrated in mitotic regulation, the substrates involved in chromosome segregation and whether HsCdc14A plays an active role in mitosis remain unclear.

HsCdc14A contains an NLS motif, a DSP domain and an NES motif. Furthermore, HsCdc14 exhibits an inhibitory self-association, in which the C-terminal domain binds and inhibits the phosphatase domain located at the N-terminus (27). Once the inhibition of C-terminus is released, the activity of the phosphatase domain, located at the N-terminus, is increased. The present study demonstrated that ZIPK interacted with the N-terminus of HsCdc14A, which contains the NLS and DSP domains. In addition, the effect of co-transfection of HEK 293T cells with ZIPK and HsCdc14A was similar to the effect of the cells transfected with the HsCdc14A N-terminus; therefore, ZIPK-mediated phosphorylation may activate the phosphatase activity of HsCdc14A. Furthermore, the results of the present study indicated that ZIPK may affect the cell cycle by interacting with the N-terminus of HsCdc14A. Notably, the effect of ZIPK and HsCdc14A overexpression on cell apoptosis was similar to the effect of ZIPK alone, but more evident than the effect of HsCdc14A alone. Apoptosis was not affected significantly more by the increased phosphatase activity of HsCdc14A along with ZIPK compared with cells transfected with $\mathrm{HsCdc14A}$ alone. Therefore, these two proteins may be associated with the same apoptotic pathway. Future experiments are required to investigate this hypothesis.

In conclusion, the results of the present study demonstrated that ZIPK may interact with the HsCdc14A protein. These findings indicated that ZIPK may also be involved in the regulation of the cell cycle. Further research regarding the regulation of HsCdc14A by ZIPK is required to provide valuable insight on the effect of ZIPK on the cell cycle. ZIPK may be a potential target candidate for the treatment of diseases associated with cell proliferation.

\section{Acknowledgements}

This study was supported by grants from the National Nature Science Foundation for Young Scientists of China (no. 30900541) and the 985 Project (no. 985III-YFX0302).

\section{References}

1. Kawai T, Matsumoto M, Takeda K, Sanjo H and Akira S: Zip kinase, a novel serine/threonine kinase which mediates apoptosis. Mol Cell Biol 18: 1642-1651, 1998.

2. Kogel D, Plottner O, Landsberg G, Christian S and Scheidtmann KH: Cloning and characterization of DLK, a novel serine/threonine kinase that is tightly associated with chromatin and phosphorylates core histones. Oncogene 17: 2645-2654, 1998.

3. Komatsu S and Ikebe M: ZIP kinase is responsible for the phosphorylation of myosin II and necessary for cell motility in mammalian fibroblasts. J Cell Biol 165: 243-254, 2004.

4. Engemann H, Heinzel V, Page G, Preuss U and Scheidtmann KH: DAP-like kinase interacts with the rat homolog of schizosaccharomyces pombe CDC5 protein, a factor involved in pre-mRNA splicing and required for $\mathrm{G} 2 / \mathrm{M}$ phase transition. Nucleic Acids Res 30: 1408-1417, 2002.

5. MacDonald JA, Borman MA, Muranyi A, Somlyo AV, Hartshorne DJ and Haystead TA: Identification of the endogenous smooth muscle myosin phosphatase-associated kinase. Proc Natl Acad Sci USA 98: 2419-2424, 2001.

6. Niiro $\mathrm{N}$ and Ikebe M: Zipper-interacting protein kinase induces $\mathrm{Ca}(2+)$-free smooth muscle contraction via myosin light chain phosphorylation. J Biol Chem 276: 29567-29574, 2001.

7. Sato N, Kawai T, Sugiyama K, et al: Physical and functional interactions between STAT3 and ZIP kinase. Int Immunol 17: 1543-1552, 2005.

8. Vetterkind S, Illenberger S, Kubicek J, et al: Binding of par-4 to the actin cytoskeleton is essential for Par-4/Dlk-mediated apoptosis. Exp Cell Res 305: 392-408, 2005.

9. Vetterkind S and Morgan KG: The pro-apoptotic protein Par-4 facilitates vascular contractility by cytoskeletal targeting of ZIPK. J Cell Mol Med 13: 887-895, 2009.

10. Li L, Ernsting BR, Wishart MJ, Lohse DL and Dixon JE: A family of putative tumor suppressors is structurally and functionally conserved in humans and yeast. J Biol Chem 272: 29403-29406, 1997.

11. Lou Y, Yao J, Zereshki A, et al: NEK2A interacts with MAD1 and possibly functions as a novel integrator of the spindle checkpoint signaling. J Biol Chem 279: 20049-20057, 2004.

12. Chen JS, Hu HY, Zhang S, et al: Brap2 facilitates HsCdc14A Lys-63 linked ubiquitin modification. Biotechnol Lett 31: 615-621, 2009.

13. Gietz RD, Schiestl RH. Applications of high efficiency lithium acetate transformation of intact yeast cells using single-stranded nucleic acids as carrier. Yeast 7: 253-63, 1991.

14. Zheng Q, Huang ZH, Tang FX, Huang ZZ, Che XY: Highly efficient construction of recombinant adenovirus containing double suicide gene driven by cytomegalovirus promoter using two-step $\mathrm{CaCl} 2$ transformation method. Di Yi Jun Yi Da Xue Xue Bao 6: 575-577, 2003 (In Chinese).

15. Zhou W, Wang X, Li L, et al: Depletion of tubulin polymerization promoting protein family member 3 suppresses HeLa cell proliferation. Mol Cell Biochem 333: 91-98, 2010.

16. Preuss U, Bierbaum H, Buchenau P and Scheidtmann KH: DAP-like kinase, a member of the death-associated protein kinase family, associates with centrosomes, centromers, and the contractile ring during mitosis. Eur J Cell Biol 82: 447-459, 2003.

17. Preuss U, Landsberg $G$ and Scheidtmann KH: Novel mitosis-specific phosphorylation of histone $\mathrm{H} 3$ at Thr11 mediated by DIK/ZIP kinase. Nucleic Acids Res 31: 878-885, 2003.

18. Hardy CF: Characterization of an essential Orc2p-associated factor that plays a role in DNA replication. Mol Cell Biol 16: 1832-1841, 1996.

19. Jaspersen SL, Charles JF, Tinker-Kulberg RL and Morgan DO: A late mitotic regulatory network controlling cyclin destruction in saccharomyces cerevisiae. Mol Biol Cell 9: 2803-2817, 1998.

20. Stegmeier F and Amon A: Closing mitosis: the functions of the Cdc14 phosphatase and its regulation. Annu Rev Genet 38: 203-232, 2004.

21. Vazquez-Novelle MD, Esteban V, Bueno A and Sacristan MP: Functional homology among human and fission yeast Cdc14 phosphatases. J Biol Chem 280: 29144-29150, 2005. 
22. Mailand N, Lukas C, Kaiser BK, Jackson PK, Bartek J and Lukas J: Deregulated human Cdc14A phosphatase disrupts centrosome separation and chromosome segregation. Nat Cell Biol 4: 317-322, 2002.

23. Kaiser BK, Zimmerman ZA, Charbonneau H and Jackson PK: Disruption of centrosome structure, chromosome segregation, and cytokinesis by misexpression of human Cdc14A phosphatase. Mol Biol Cell 13: 2289-2300, 2002.

24. Gruneberg U, Neef R, Honda R, Nigg EA and Barr FA: Relocation of Aurora B from centromeres to the central spindle at the metaphase to anaphase transition requires MKlp2. J Cell Biol 166: 167-172, 2004.
25. Paulsen MT, Starks AM, Derheimer FA, et al: The p53-targeting human phosphatase hCdc14A interacts with the Cdk1/cyclin B complex and is differentially expressed in human cancers. Mol Cancer 5: 25, 2006.

26. Bembenek J and Yu H: Regulation of the anaphase-promoting complex by the dual specificity phosphatase human Cdc14A. J Biol Chem 276: 48237-48242, 2001.

27. Yuan K, Hu H, Guo Z, et al: Phospho-regulation of HsCdc14A by polo-like kinase 1 is essential for mitotic progression. J Biol Chem 282: 27414-27423, 2007. 